\title{
Bringing Homologies Into Focus
}

\author{
Anastasia Thanukos
}

Published online: 26 September 2008

(C) Springer Science + Business Media, LLC 2008
Subsequent evolution in the lineage leading to modern humans and in the lineage leading to modern lizards has resulted in differences between our two appendages: bones with slightly different shapes, orientations, and functions. Nevertheless, the common evolutionary origin of the two appendages is evident in their deep similarities. Though differently shaped, corresponding bones are present in the two groups of organisms and develop in similar ways. The same set of arguments applies to human and lizard eyes. Both lineages bear the same type of complex lens eyes that we inherited from our common ancestor (Fig. 2).

Analogies, on the other hand, are similar traits that evolved through convergent evolution. In the classic example, evolution independently shaped the forelimb of a dinosaur, the forelimb of an ancient mammal, and the gilllike appendage of an ancient insect into wings that could carry their bearers (birds, bats, and insects) through the air. The fact that modern birds, bats, and most insects have wings reflects, not common ancestry, but similar processes of exaptation and subsequent adaptation. In the same way, the complex lens eyes of humans and squid are remarkably similar (Fig. 3) but analogous, since they evolved independently in our ancestral lineages (Fig. 4).

\section{Beyond Analogies}

So far, so good. This is the view presented by standard biology textbooks. However, biologists often depart from high school texts in their analysis of analogies (e.g., Hall 2007; Gregory 2008). The counterpart of homology is usually considered to be homoplasy, a much broader concept than analogy. A homoplasious trait is a similarity among organisms that was not inherited from the common ancestor of those organisms. Homoplasies can evolve in 
Fig. 1 Humans and lizards inherited appendages with similar structures from a common ancestor whose limbs also had this structure. Illustration adapted with permission from the Understanding

Evolution website

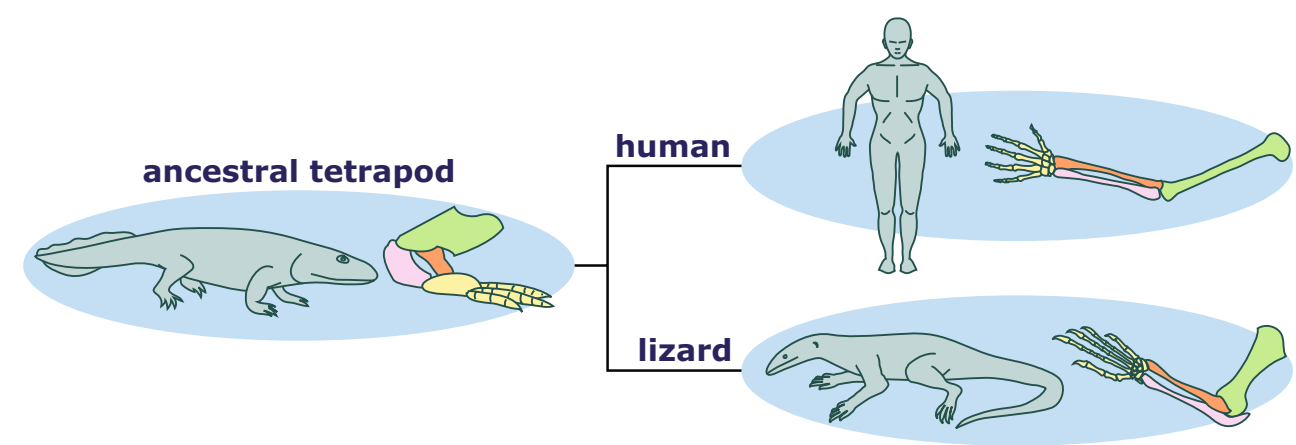

three ways (though the lines between these categories are often blurry):

- Convergent evolution. This process produces analogies, as discussed above. Two lineages that begin with different traits evolve a similar characteristic independently of one another, often because both lineages face similar environmental challenges and selective pressures. For example, two distantly related plant lineages might evolve analogous tubular red flowers under selection from hummingbird nectar feeders. Or, as in Fig. 5a, fish lineages that originally had different body patterns might independently evolve analogous vertical stripes, perhaps because of selection for a particular camouflage pattern.

- Parallel evolution. In this process, two traits that are already similar (usually because of common ancestry) independently evolve the same set of changes - generally meaning that the same set of underlying genes are involved. This is illustrated in Fig. 5b in which two closely related fish lineages evolve in the same way, resulting in two lineages with a striped body pattern. A real-life example of parallel evolution also involves fish. The ancestral stickleback fish was marine-living and heavily armored by sturdy plates. However, several stickleback lineages invaded new, freshwater environments. Many of these now-freshwater lineages independently evolved the same sort of genetic changes which produce a lightly armored body form. Scientists are not sure why the lightly armored trait was favored in so many different lineages, but it may have involved selection for increased body flexibility (Colosimo et al. 2005).

- Evolutionary reversal. In this process, a lineage evolves toward one of its ancestral traits, effectively losing a more recently evolved trait. This is generally thought to involve genetically "reactivating" the ancestral trait (e.g., see Marshall et al. 1994). If a related lineage has retained the ancestral trait, the two lineages will share a similar feature, not because of inheritance from a common ancestor but because of an evolutionary reversal in one lineage. This is illustrated in Fig. $5 \mathrm{c}$ in which an originally striped fish lineage loses its stripes and then regains them, producing a species similar to a closely related lineage that never lost its stripes in the first place. A real-life example of evolutionary reversal involves stick insects, which evolved from a winged ancestor but then lost those wings. Stick insects diversified into many different species in their new wingless form. However, a few stick insect lineages seem to have independently "reevolved" wings by reactivating an ancient genetic program that produces wings (Whiting et al. 2003). These newly winged insects now have wings similar to one another and to more distantly related winged insects - insects which never lost their wings in the first place. However, the wings in these different groups are homoplasious since they were not directly inherited from a common ancestor: the winged stick insects went through an intermediary wingless stage.

Eye evolution, in fact, exhibits both convergent and parallel evolution. The similarity in structure between squid and vertebrate eyes (Fig. 3) is a classic example of convergent evolution, since our most recent common ancestor may have borne nothing more complex than a few light-sensitive cells. In a remarkable example of parallel evolution, the same protein (known as zetacrystallin) appears to have been independently recruited to go to work as part of the lens in two distantly related groups of vertebrates: the llama and guinea pig families (Gonzalez et al. 1995). The zeta-crystallin in the lenses of these relatively remote relatives represents the legacy of parallel

Fig. 2 Humans and lizards inherited their complex, lensbased eye from a common ancestor that also had this sort of eye structure

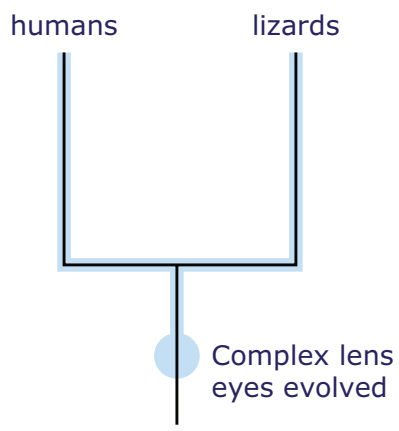


Fig. 3 Human and squid eyes are structured similarly but are analogous since they evolved independently in the two lineages. Illustration adapted with permission from the Understanding Evolution website

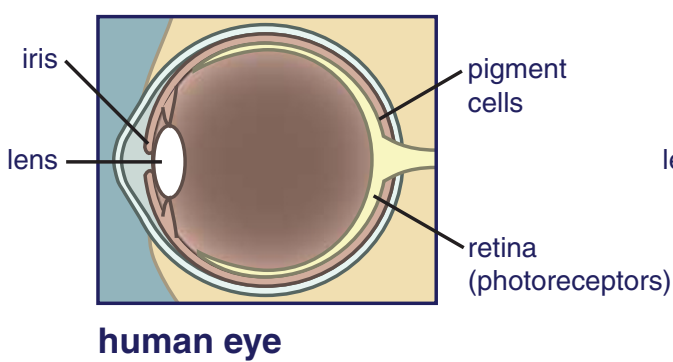

evolutionary events, not recent common ancestry. These processes, as well as evolutionary reversal, can produce traits that are strikingly similar-though not inherited directly from a common ancestor.

\section{Homologies at Many Levels}

An examination of eye evolution also highlights another, less familiar aspect of homology. Though we tend to think of homologies in terms of anatomy (e.g., the tetrapod limb, insect wing, or vertebrate eye), any heritable traitanything that can be directly or indirectly encoded in DNA - can be a homology. Coding and noncoding DNA sequences, the proteins and gene regulation systems encoded by DNA sequences, simple traits (like eye color), components of complex organs (like the lens of an eye), entire complex structures, and even behaviors (like providing parental care to offspring) can be examined to determine whether they are homologous or homoplasious among different lineages.
Partly because of this hierarchy, meaningful statements about homology must include several details:

1. Which organisms are being compared? The complex lens eye is homologous among humans, lizards, and fish, but the same trait is homoplasious between humans and squid, having evolved independently in vertebrates and mollusks. Simply identifying a trait as homologous or homoplasious is meaningless unless we know which lineages are being considered.

2. What specific aspect of the trait is being compared? Taking another example from eye evolution, we need to know whether we are considering the entirety of a complex structure (e.g., a lens-type eye), the lens itself, the proteins that make up that lens, the genes that encode those proteins, or the genetic triggers that cause those genes to be turned on in developing eyes. Each aspect of this trait may have a slightly different evolutionary story. Vertebrates, for example, have homologous, lens-based eyes, though some of the crystallin proteins that make up those lenses exhibit
Fig. 4 Humans' and lizards' complex, lens-based eyes are homologous to one another, but analogous to those of the squid

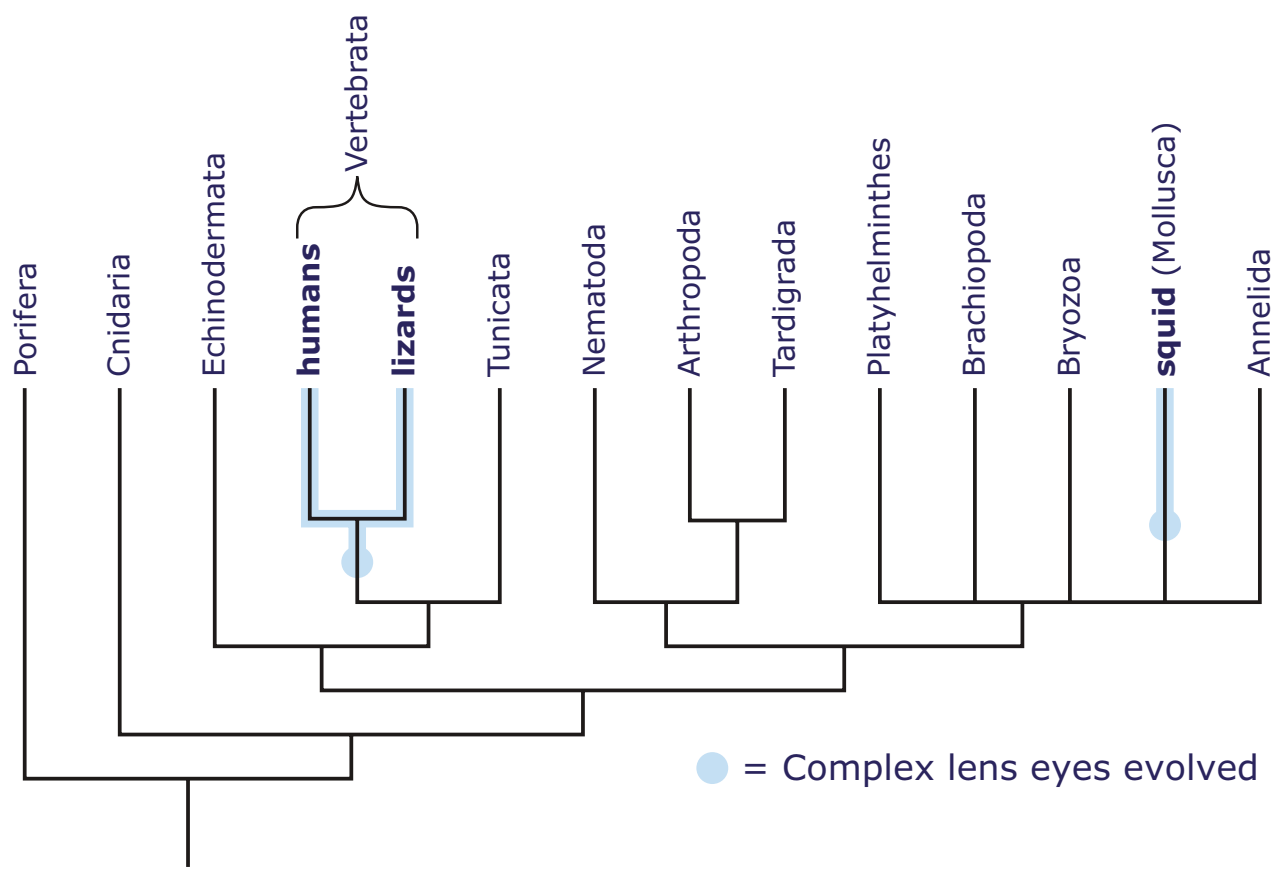


Fig. 5 Hypothetical examples of three different categories of homoplasy, as well as homology. a Striping in the two descendent fish lineages illustrated evolved through convergent evolution. b Striping in the two descendent fish lineages involves the same set of underlying genes and evolved through parallel evolution. c Both descendent fish lineages bear stripes, but one of the lineages wound up with stripes through an evolutionary reversal. d Striping in the descendent fish lineages was inherited from a common ancestor and is homologous

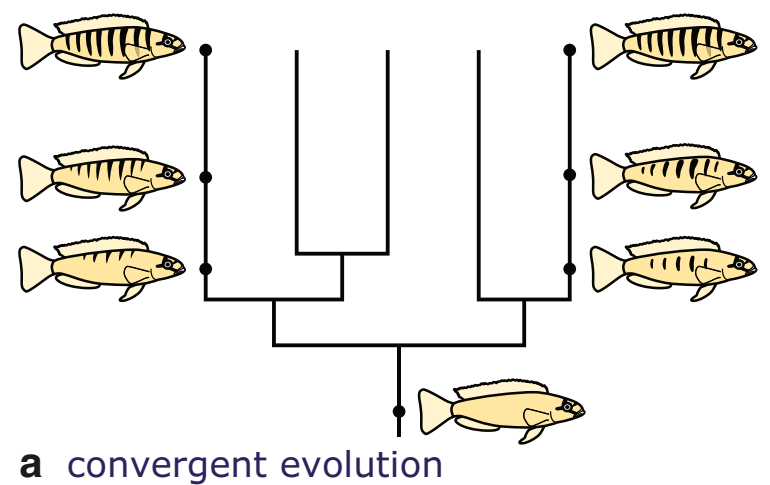

b parallel evolution
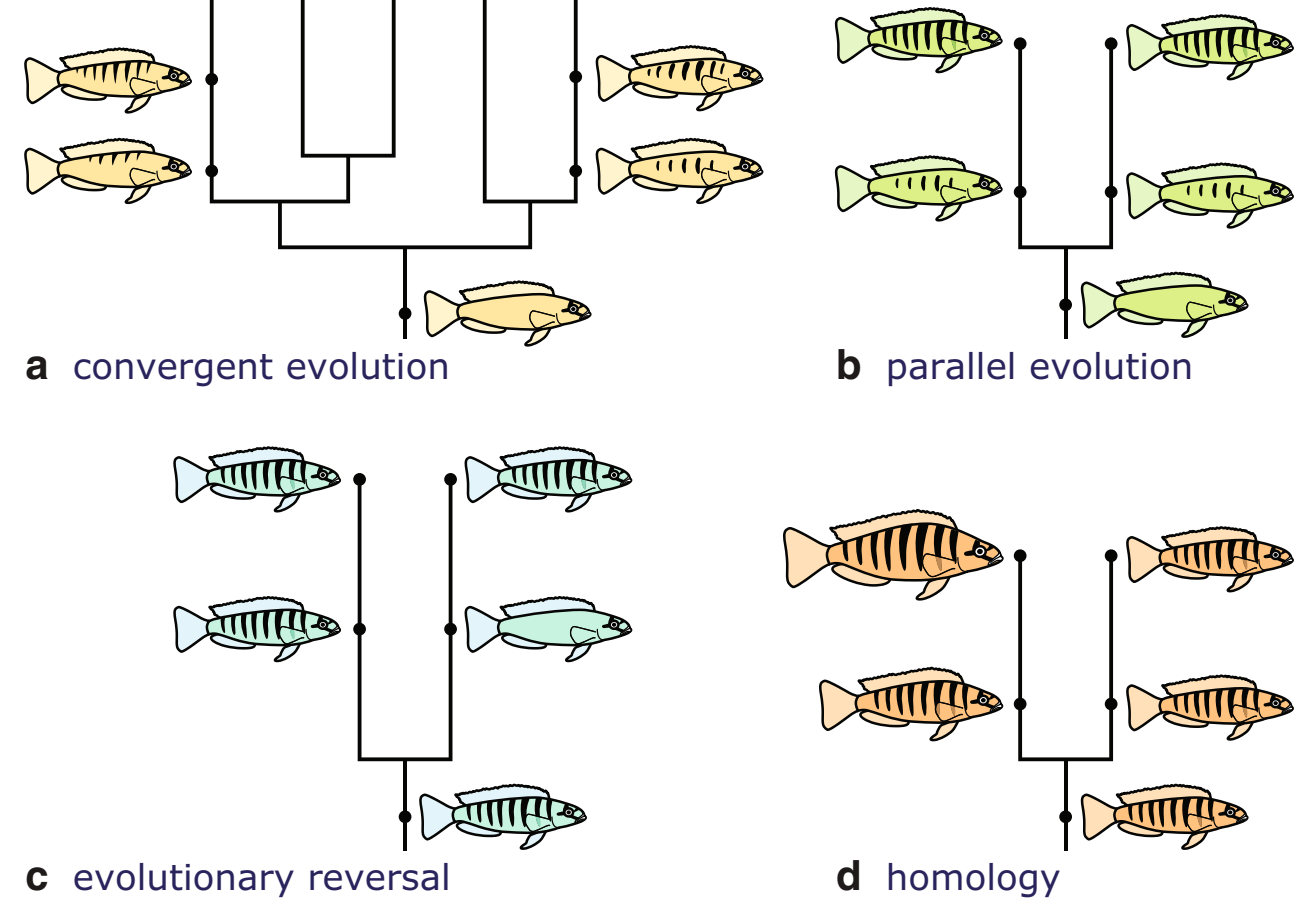

homoplasy. Different proteins have been recruited at different points in our evolutionary history to build vertebrate lenses.

3. Does the function of the trait matter? To make matters even more complex, analyses of homology may also need to consider the function of the trait. Zeta-crystallin, described above, provides a striking example. This molecule is present in modern llamas and guinea pigsand appears to have been inherited from their common ancestor, making the molecule itself homologous in these two groups. However, in that ancient common ancestor, zeta-crystallin likely performed the job of catalyzing chemical reactions and did not form part of the lens, as it now does in llamas and guinea pigs-making zetacrystallin homoplasious as a lens component in these two groups (Gonzalez et al. 1995).

Consider the evolutionary history of Pax6, which exemplifies many of these details. Pax6 is a gene that helps control what other genes do. It encodes a special type of protein known as a transcription factor. This protein can invade the nucleus of a cell, bind the DNA there, and turn other genes in that DNA off and on. Such regulatory genes can be enormously powerful, as they can set off a whole cascade of other gene actions.

Versions of Pax6 are found in almost all modern animals, suggesting that the gene is ancient-more than 500 million years old! - and that homologous versions of the gene have been inherited by many different modern animal groups from this common ancestor (Fig. 6). It is not clear what the Pax6 gene did in this ancient animal. It may have helped build the simplest type of two-cell eye or a light-sensitive protein. Or, in a case of parallel evolution, Pax6 may have originally performed a job unrelated to light sensing, but was later recruited -in several different early lineages - for the job of helping guide the development of simple visual organs. So while the Pax6 gene itself is certainly homologous among modern animals, Pax6 functioning as a visual control gene may, in fact, be homoplasious among modern animals.

Sometime after Pax6 was recruited for visual controlwhether it happened just once or several times-different lineages that inherited that gene began to evolve in slightly different directions. Some retained simple eyes. Others experienced selection for increased visual acuity and evolved elaborations on the basic form. In some lineages (e.g., vertebrates and cephalopods), evolution shaped available components into remarkably similar complex eyes (Fig. 7). In this way, animals evolved eyes that, while homoplasious as complex organs, are built from some homologous cell types and are partly controlled by a homologous gene, Pax6.

\section{Conclusion}

Through an examination of eye evolution, we have seen that distinguishing between homology and homoplasy can 
Fig. 6 The Pax6 gene itself evolved early in animal evolution and is homologous among these lineages

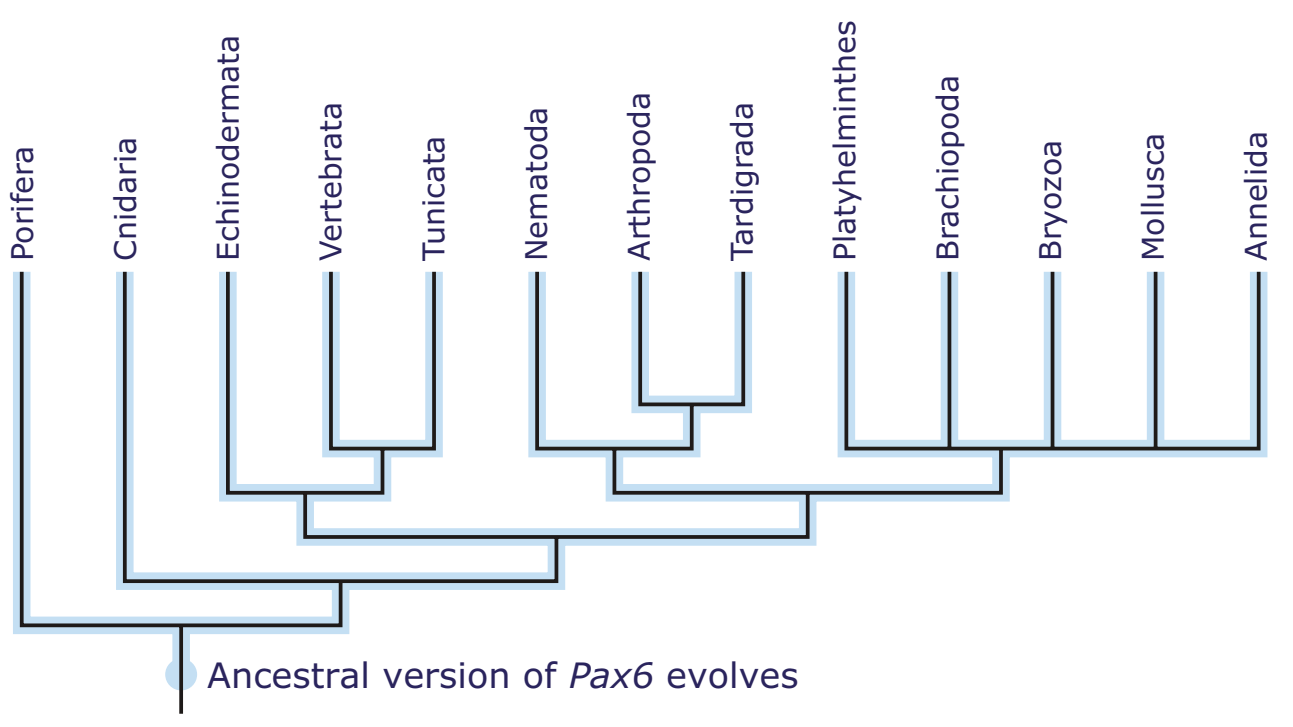

be a tricky and context-dependent business. In fact, these concepts may represent the ends of a continuum more than opposite ideas (Hall 2007). Making even a general differentiation between them depends on specifying the organisms of interest, the aspect of the trait being compared, and even the function of the trait at various points in its history. Homoplasy, especially if it arises through parallel evolution or reversals, may be difficult to detect. Nevertheless, examining these issues for each level of organization of a complex organ (from regulatory genes up to the functioning structure) provides a more accurate and complete understanding of sometimes complex evolutionary histories and helps us understand how sophisticated and highly interdependent organs can evolve from humble beginnings. Especially when it comes to the evolution of eyes, the concepts of homology and homoplasy may not be simplistic, but they are illuminating.

\section{Give Me an Example of That}

Want more examples of homologies and homoplasies? Check these out:

- Homologies. We have seen that homologies are simply traits inherited from a common ancestor. However, not all homologies are obvious. If two homologous structures have been adapted for different roles, they may not look very much alike. For example, though they are homologous structures, the chomping front teeth of a beaver look quite different from the tusks of an elephant. Nevertheless, once you know what homologies are, you can find them almost anywhere. This short article from the Understanding Evolution website takes a look at five examples of homology, including structural, genetic, and behavioral examples: http:// evolution.berkeley.edu/evolibrary/article/homology_01.
Fig. 7 Pax6 may have been recruited for roles in eye development in different lineages at different points in time. Different sorts of complex eyes later evolved in different lineages, exapting many different genetic and anatomical components

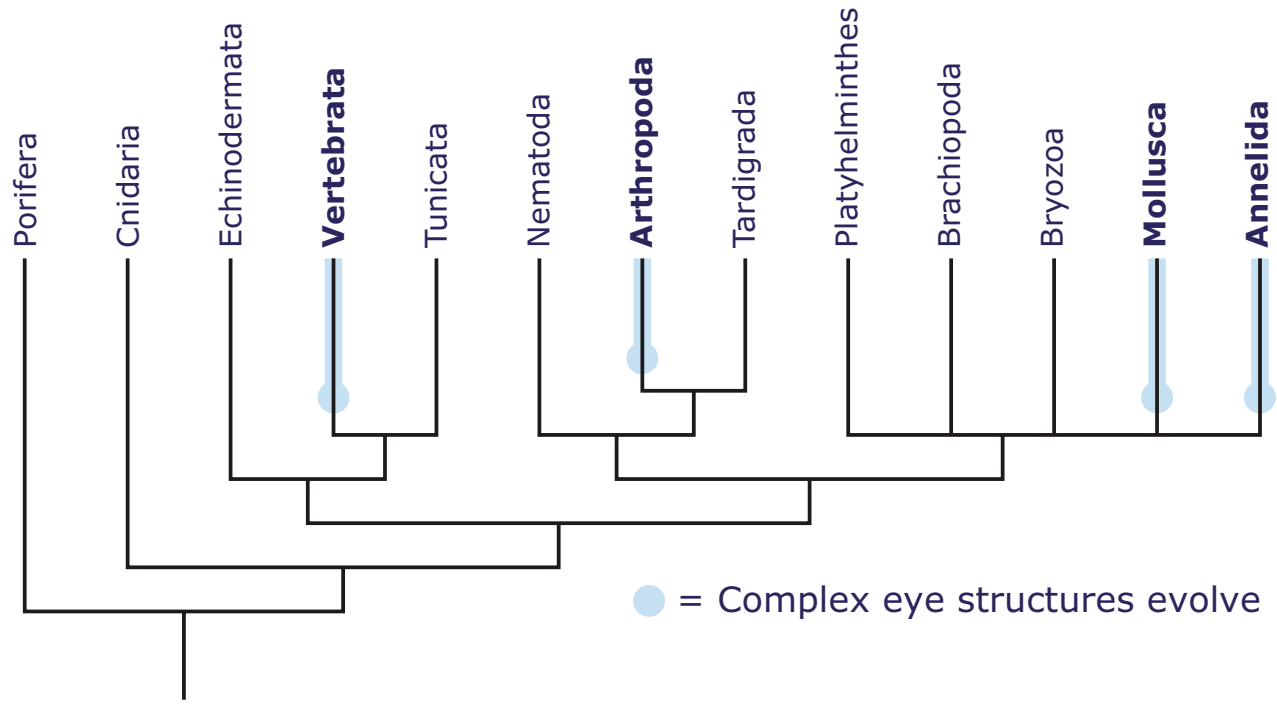


- Analogies. As we have seen, analogies are one important category of homoplasy. They often result when two species face a similar problem or challenge and evolution shapes both lineages in similar ways. This short article from the Understanding Evolution website takes a look at six examples of structural analogy: http://evolution. berkeley.edu/evolibrary/article/analogy_01.

\section{Branch Out}

Many articles in this issue explore the evolution of complex organs, like eyes. Genetics-especially developmental genetics - has played a key role in this process. Explore the following online resources to learn more about this evolution and developmental genetics:

- Review basic concepts from the field of evo-devo in this advanced tutorial from the Understanding Evolution website: http://evolution.berkeley.edu/evolibrary/ article/evodevo_01.

- Learn how developmental genetics is reshaping our understanding of evolution in this article from National Geographic: http:/ngm.nationalgeographic.com/ngm/ 0611/feature4/index.html.

- Expand your understanding of developmental genetics with this video from Howard Hughes Medical Institute. In lecture 4 of a four-part series entitled From Butterflies to Humans, evolutionary biologist Sean Carroll uses the developmental genetics of insects to explain how old genes can learn new tricks and how this can help us understand human evolution: http:// www.hhmi.org/biointeractive/evolution/lectures.html.

\section{Dig Deeper}

Visit Understanding Evolution online to find out even more about some of the concepts addressed here.

- An interactive web feature on the evolution of eyes: http://evolution.berkeley.edu/evolibrary/article/eyes_01.

\section{In the Classroom}

Homology and homoplasy can be taught at many grade levels. While older students may be prepared to grapple with the subtleties described in this article, junior high and grade school students can certainly understand the basics of these concepts. You might begin to introduce the concepts with one of these interactive online modules:

- Similarities and differences: Understanding homology and analogy for grades 6-8, http://evolution.berkeley. edu/evolibrary/article/similarity_ms_01.
- Similarities and differences: Understanding homology and analogy for grades 9-12, http://evolution.berkeley. edu/evolibrary/article/similarity_hs_01.

Older students can extend their learning of these concepts with the following lessons and activities:

- Comparison of human and chimp chromosomes. A lesson dealing with homologies at the molecular level from the Evolution and the Nature of Science Institute for grades 9-12. In this lesson, students observe that the banding patterns seen on stained chromosomes from humans and chimpanzees show striking similarities. Possible evolutionary relationships are explored, as are the chromosomes and relationships of other apes: http:// www.indiana.edu/ ensiweb/lessons/chromcom.html.

- Using trees to understand plants. An article with discussion questions from the Understanding Evolution website for grades 9-12. This research profile follows scientist Chelsea Specht as she uses phylogenies to investigate the pattern of homology and homoplasy in the evolutionary history of tropical plants and their pollinators - and in the process, tries to figure out how to conserve endangered species: http://evolution.berkeley. edu/evolibrary/article/specht_01.

- The new shrew that's not. A short news article with discussion questions, links to lessons, and a podcast from the Understanding Evolution website and the National Evolutionary Synthesis Center for grades 912. This news brief from March of 2008 describes scientists' discovery of a new mammal species, a giant elephant shrew. Though elephant shrews share many analogous traits with regular shrews, genetic homologies suggests that elephant shrews actually sprang from a much older (and perhaps more charismatic) branch of the tree of life - the one belonging to elephants and their relatives: http://evolution.berkeley.edu/evolibrary/ news/080301_elephantshrew.

Acknowledgements The author wishes to thank T. Ryan Gregory, Todd Oakley, and Judy Scotchmoor for the helpful comments on earlier drafts.

\section{References}

Colosimo PF, Hosemann KE, Balabhadra S, Villarreal G Jr, Dickson $\mathrm{M}$, Grimwood J, et al. Widespread parallel evolution in sticklebacks by repeated fixation of Ectodysplasin alleles. Science 2005;307:1928-33. doi:10.1126/science.1107239.

Gonzalez P, Rao PV, Nunez SB, Zigler JS Jr. Evidence for independent recruitment of $\zeta$-crystallin/quinone reductase (CRYZ) as a crystallin in camelids and hystricomorph rodents. Mol Biol Evol 1995;12:773-81. 
Gregory, TR. The evolution of complex organs. Evolution: Education and Outreach 2008;1. doi:10.1007/s12052-008-0076-1

Hall BK. Homoplasy and homology: dichotomy or continuum? J Hum Evol 2007;52:473-9. doi:10.1016/j.jhevol.2006.11. 010

Marshall CR, Raff EC, Raff RA. Dollo's law and the death and resurrection of genes. Proc Natl Acad Sci U S A 1994;91:122837. doi:10.1073/pnas.91.25.12283.
Oakley, TH., Pankey, MS. Opening the "black box": the genetic and biochemical basis of eye evolution. Evolution: Education and Outreach 2008;1. doi:10.1007/s12052-008-0090-3

Piatigorsky, J. A genetic perspective on eye evolution: gene sharing, convergence and parallelism. Evolution: Education and Outreach 2008;1. doi:10.1007/s12052-008-0077-0

Whiting MF, Bradler S, Maxwell T. Loss and recovery of wings in stick insects. Nature 2003;421:264-7. doi:10.1038/nature01313. 